\title{
DNA Nanodevices Confined on DNA Origamis: an Isothermal Ratchet Driven by Boundary Conditions
}

\author{
Jean-Pierre Aime, Juan Elezgaray and Oscar Mendoza \\ VIBBnano,CBMN Ave Geoffroy St Hilaire CNRS University Bordeaux Pessac 33600, France \\ Email: jp.aime@cnanogso.org
}

\begin{abstract}
In this note we investigate the diffusive behaviour and the boundary conditions of DNA nanodevices using the toe hold mediated strand displacement method. The goal is to extract the basic principles governing the difference observed in diluted solution and in confined environment where the devices are tethered on a DNA Origami. We note that the excluded volume interaction between the two strands running in opposite direction must give a sub diffusive behaviour that can lead to very long waiting times between jumps. When the Boundary Conditions generate a strong asymmetry in the device, the probability to perform the logical operation, thus to remove the output strand, is one. However, we envision unexpected marked differences between diluted solutions and confined environment both for controlling the boundary condition and the sub diffusive behaviour. These differences rise new questions on the interest to use the toe hold mediated strand displacement method on DNA Origamis.
\end{abstract}

Keywords: DNA Nanotechnology, confined chemical reactions, DNA logical gate, DNA origami, modular algorithm.

\section{Introduction}

DNA strands are key building units that boost and create innovative routes in the domain of nanotechnology. Over the last three decades, the use of DNA strands has lead to several remarkable achievements. Triggered by the Seeman vision in the early eighties [1,2], which we can shortly summarised as follow: the use of DNA molecules must not be restricted to its primary genetic code function but used as any kind of nanomaterial. Since then, many works have contributed to establishing firm strategies based on DNA to conceive and fabricate a wealth of structures and functions. Among them, the most significant are the folding of origami [3] and the DNA motor devices [4] that turn out to be fertile enough to design networks of chemical reactions with a constructed rational approach $[5,6,7,8,9,10,11,12]$. The combination of DNA origami and DNA motor devices gives the great opportunity to elaborate modular Boolean algorithm based on ordered sequences of chemical reactions confined in an almost perfect topological arrangement $[13,14,15,16,17]$. Numerous applications are envisioned especially those addressing nano-medecine and environment $[18,19]$.

One key feature motivating the present work is that life systems have to select molecules and ensure robust functions in a crowded environment. This happens almost at any step, at the primary level of gene expression with the appropriate selection of promoters in the network of transcription factors, in the ribosomal machinery to select the right amino acid, in immunology where the $\mathrm{T}$ cells have to select the pathogen among a large population of molecules in order not to kill the healthy cells, in an efficient transfer of hormones and neurotransmitters to cells or synapse connections. Life systems have developed numerous strategies like kinetics proofreading with square rate discrimination, delay time of integration, use of selected bandwidth in a cascade of enzymatic reactions upon the action of a signal molecule, allosteric functions and so on [20,21]. One interesting feature is the noticeable difference between hormones and neurotransmitters. Both have the same kind of action, but, as quoted by Kandel and al [22], the main difference is that hormones diffuse at random over the cells while neurotransmitters are confined in space at the synapse connection, which makes the flow of information much more efficient. The effect of the confinement is not understood, in particular the role of the fluctuations. Therefore, the study of chemical reactions in confined space where the topological constraints are well set is of the utmost interest. Beyond this philosophical introduction, there are growing technical efforts and new experiments 
that DNA nanotechnology affords. This is what we discuss here, our bottom line being a reductionist approach that aims to extract some key features.

The important factor is the algorithmic structure with which the DNA structure and functions are designed, those algorithms being based on the Watson-Crick (WC) pairing rules. Obviously, the use of a simple algorithm allowing to conceive and to fabricate material structures and functions is in itself a great progress. However, DNA strands are flexible polymers that don't exactly behave as simple bits $(0,1)$ with which one would have been allowed to describe a cascade of chemical reactions using simple Shannon rules. Even short DNA strands are quite complex objects made of a huge number of degrees of freedom that can exhibit a great variety of conformational fluctuations. In this respect, the confinement of interacting DNA strands creates new situations in which the fluctuations can play a leading role. The confinement of chemical reactions provides several advantages; one is to restrict the flow of leaks and unwanted cross talks; a second is to increase the speed of the computation. In both cases, these two significant advantages increase the capacity and complexity of the computation and its robustness. To exploite those advantages, we recently did work using DNA nanodevices tethered on origami templates [23] using the toe hold strand displacement technique $[24,25,26]$. While it is a convenient way to construct modular Boolean operations with a sequence of chemical reactions, it happens that the use of the strand displacement in diluted and confined environment rises new questions.

The present work is an attempt to evaluate the gain we may have when the DNA nanodevices are confined on DNA Origamis. The ratchet function of the DNA nanodevice is driven by the boundary conditions and, because the whole process is a complex one, we divide the chemical reaction in three steps. A first step in which the toe hold of the input binds the template of the gate; a second step is focused on the competition between the input and the output strands that leads to an anomalous diffusion behaviour and a third step shows how the boundary conditions can modify the kinetics competition between the input and the output strands. The paper is organised as follow: in the first paragraph we introduce the basic principles we need, in the second paragraph we discuss first the single file problem applied to the DNA nanodevices then the different types of boundary conditions that can be engineered in diluted solutions and when a DNA origami is used. Finally, we discuss the effect of the boundary conditions upon the probability for the output to be removed from the gate.

\section{Basic Principles}

\subsection{DNA Strand Displacement a Random Walk with Excluded Volume Interaction between Walkers}

We have to deal with a biased random walk with an excluded volume interaction between particles, where the particles are not simple beads but flexible short chains. This is a complex problem and the reason why we adopt a reductionist approach, with which we hope we could extract some basic principles. Based on the numerous previous works $[24,25,26]$ and our recent experimental results and analysis investigating the behaviour of DNA nanodevices on origamis [23,27], the present work is an attempt to improve our understanding of the head motion of a single strand pushing and removing another almost identical one that already forms a duplex with its complementary single strand. Such a chemical reaction can be seen as a race competition between two identical, or slightly different runners, running in opposite direction along a 1 dimensional energy landscape.

In the simplest case where no competition takes place, it is commonly accepted that the two strands DNA hybridisation behaves as a Markov process. For short strands, the average square excursion of the head of the duplex formation is given by a random walk (see the simple picture displayed in figure 1) and the diffusion law at long time is :

$$
\left\langle L^{2}\right\rangle=D_{e f f} \tau
$$

where $D_{\text {eff }}$ is an effective diffusion constant accounting for the random walk process. As a consequence the time needed to move up to a length $\mathrm{L}$ corresponding to $\mathrm{N}$ sites scales as:

$$
\tau=N^{2} \tau_{K}
$$

where $\tau_{K}$ is the Kramer escape time [27]. 

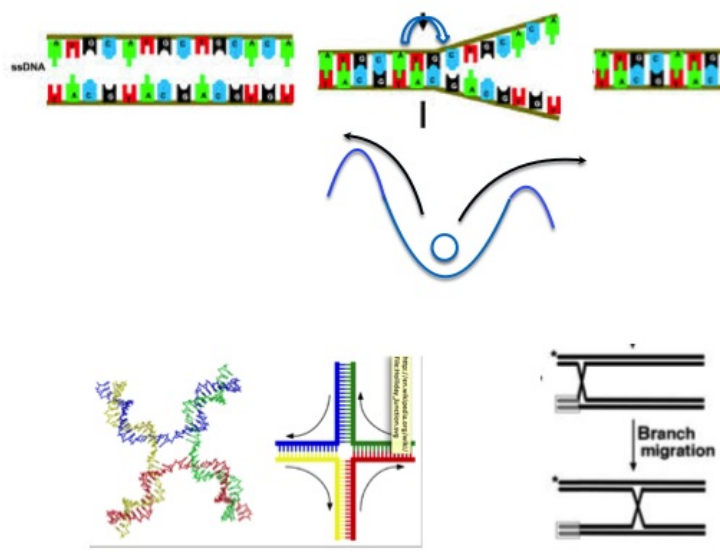

Figure 1. (a) Sketch of the hybridisation of two complementary single strands. The motion of the head of the hybridisation process can be described as the hoping of a bead out of a potential well. The hoping time is an exponential function of the barrier height and is given by the Kramer's rule. (b) Holiday junction and scheme of the branch migration. The branch migration can take a much longer time, several orders of magnitude, than that of a simple hybridisation process.

When a strand displacement takes place, this simple picture is no longer a correct one, the two strands walking along the same track collide each other (see figure 2). The interaction between the two particles affects the Brownian diffusion. The effect of the interaction between Brownian particles has been for long studied with the central question asking whether the law or else only the diffusion constant is modified. One can intuitively understand that at two or three dimension an excluded volume interaction between particles would not modify the diffusion law. At two or three dimension, the particle will always have paths to avoid the occupied sites so that the usual diffusion law at long time still applies. It is also obvious that at one dimension it becomes a complete different matter, the excluded volume interaction and the collisions will have a dramatic effect. At one dimension a particle cannot overpass another particle neither can move into an already occupied site. Leaving aside prefactors such as the dependence on the fraction of the occupied sites, at long time or for large excursion the diffusion law is modified with a significant slow down leading to a sub-diffusion behaviour $[28,29]$ :

$$
\left\langle L^{2}\right\rangle=D_{e f f} \tau^{1 / 2}
$$

or else

$$
\sqrt{\left\langle L^{2}\right\rangle}=\sqrt{D_{e f f}} \tau^{1 / 4}
$$

which gives

$$
\tau=N^{4} \tau_{K}
$$

The overall time spent to displace a strand becomes much greater than the one needed to propagate a simple hybridisation process. For practical purpose let's start with a Kramer escape time $\tau_{K}$ of $10^{-5}-10^{-6}$ s. The Kramer escape time $\tau_{K}$ gives the order of magnitude of the hoping rate for a particle located in a site to either escape to the left or to the right nearby sites. For a strand displacement of length 10 to 20 bp, e.g. $\mathrm{N}=10$ to 20 sites, the experimental results give a typical migration time of about $\tau=1$ second [24]. Therefore, the chemical reaction that removes the displaced strand from its track is achieved within a time $\tau=\mathrm{M} \tau_{K}$, where $\mathrm{M}$ is the total number of attempts of left and right jumps between neighbouring sites. With $\tau=1$ second, we obtain $\mathrm{M}=10^{5}-10^{6}$. With a number of sites $\mathrm{N}=20$, the number of jumps $\mathrm{M}$ we calculate is roughly the value we get with a $N^{4}$ law dependence. Despite the fact that everything 
happens at a molecular level with a set of small barrier heights of only a few $\mathrm{k}_{B} \mathrm{~T}$, this unusual very long time must be a typical value when two strands walk along the same track in opposite direction.

\subsection{Contact Events and Flow of Output Strands}

The study of a Brownian motion, in which a steric interaction between particles becomes a relevant process, is a difficult task. It is even worse when we have to consider the competition between two flexible single strands walking on the same track in opposite direction. Therefore, our main purpose is to find a method that evidences the difference in the chemical reaction paths when the chemical reactions are performed in diluted solutions and in a confined environment. A sketch of the chemical reaction involving a toehold DNA strand displacement is displayed in figure 2. In addition, the use of a DNA origami as a template for the DNA devices contains in itself new parameters that increases the complexity of the system. As a consequence, it is worth dividing the overall chemical reaction in several elementary steps, each of them being as simple as possible (see figure 3 ).

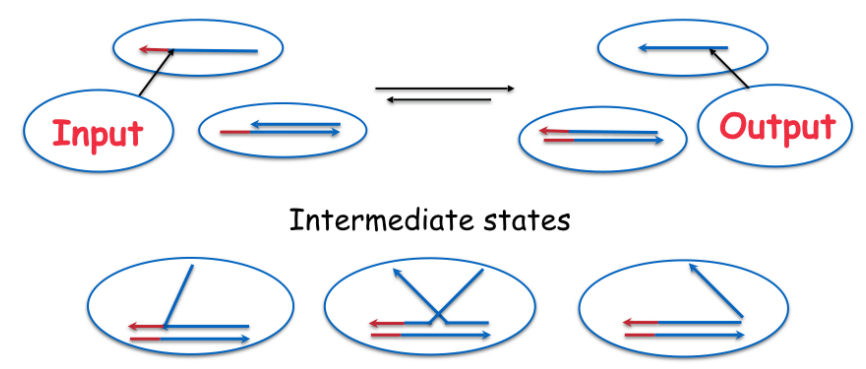

Figure 2. Sketch of a chemical reaction of a DNA nano device based on the forward motion of a DNA strand initiated by a toehold DNA sequence (input). Input with its toehold (red) and common sequence with the output (blue) that displaces the output strand. Possible intermediate states are sketched.

First, one has to calculate the probability per unit time and per mole that the input strand hits the target. This rate gives an upper bound of the bimolecular rate constant $\mathrm{k}_{\text {on }}$. Once the input strand has hit the target, question rises about its ability to perform the whole chemical reaction. The product of the chemical reaction is an output strand, which means the hybridisation of the toe hold must be stable enough in order to be able to initiate the competition between the input and the output strands running on the same 1-Dimensional track. This latter process is where the difficulty rises. A simplified approach that may grasp some of the important features is to describe the whole process as an isothermal ratchet and to use a Fokker Planck Equation (FPE). We showed recently that the FPE is a useful tool for this stochastic problem, especially when one wants to evaluate the effect of the boundary conditions and of the asymmetry of the device [27].

In diluted solution, the rate of hitting will be governed by a diffusion process and is given by:

$$
k_{o n}^{\text {diff }}=D \phi N_{a v}=10^{8} M^{-1} s^{-1}
$$

with $\mathrm{D}=10^{-5} \mathrm{~cm}^{2} / \mathrm{s}$ the diffusion constant, $\phi=10 \mathrm{~nm}$ the size of the molecule and $N_{a v}$ the Avogadro number. This rate gives an upper bound value in diluted solution, which is about two orders of magnitude greater than those given by the experiments $k_{\text {on }}^{e x p}=10^{6} M^{-1} s^{-1}$. This large difference means that we need several nucleotides to form a stable duplex in order to ensure that the collisions between complementary strands are able to initiate the chemical reaction. There is a balance between the rate of collisions at a given concentration c of reactant $\mathrm{D} \phi \mathrm{c}$ and the dissociation rate. The rate of dissociation of one nucleotide scales as the reciprocal of the Kramer's escape time, which gives $k_{\text {dissoc }}^{1}=10^{5}-10^{6} s^{-1}$, and with $\mathrm{n}$ nucleotides the rate of dissociation decreases as $k_{d i s s o c}^{n}=10^{6} s^{-1} \exp ^{-(n-1) U_{l} / k_{B} T}$, where $U_{l}=3 k_{B} T$ is the 


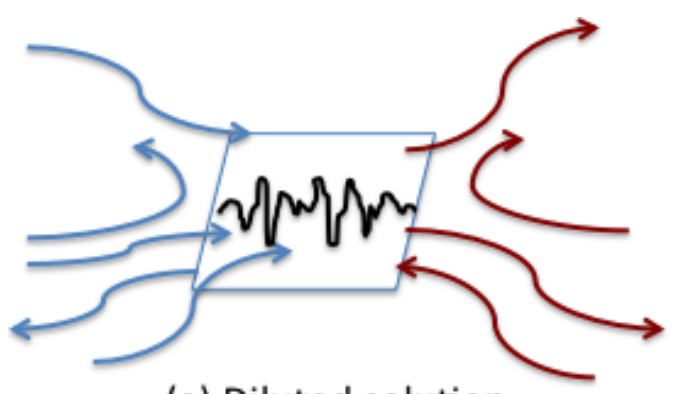

(a) Diluted solution

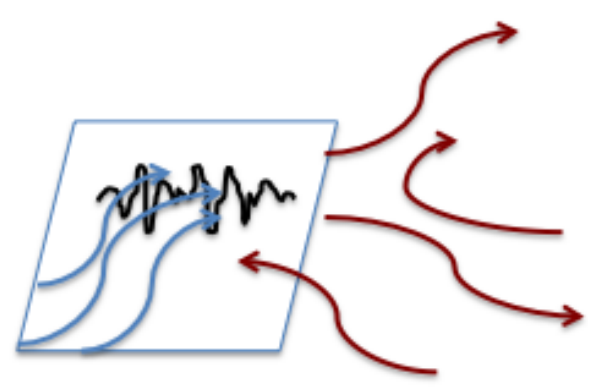

(b) Confined on origamis

Figure 3. Sketch of the three steps describing the toe hold strand displacement in diluted and confined environment. The gate (or target) is represented with a parallelogram. 1-The blue arrows mimic the Brownian motion (in diluted solution) of the input strand hitting the target. 2-The wavy black line represents an energy landscape on the gate with disordered barrier heights between sites generated by the collisions between strands. 3- The red arrows mimic the Brownian motion of the output strands when it is removed from the gate. (a) In diluted solution both strands can diffuse away from the target. (b) In confined structures the input strand is tethered on the origami and is forced to go back on the target.

binding energy of one nucleotide. Therefore, with 3-4 nucleotides the dissociation rate decreases by two orders of magnitude. These dissociation rates have to be compared with the rate of collisions without which nothing can happen. Let's consider a box of molecular size $\phi=10 \mathrm{~nm}$, with a concentration $\mathrm{c}=10 \mathrm{nM}$ the mean number of molecules flowing through the box within one second is $\mathrm{N}=\mathrm{D} \phi \mathrm{c}=60 \mathrm{~s}^{-1}$. But the target itself is also a random variable passing through the same box with the same distribution and, saying that the collision does not bear any memory effect at least for time greater than $10^{-6} \mathrm{~s}$, we get the result that the total number of collisions in the box is $N^{2}=3600$ per second, which falls within the rate of dissociation for 3-4 nucleotides. In other words, when the two strands collide the hybridisation rate of 3-4 nucleotides just balances the dissociation rate. Let us assume that each contact bears a probability close to one that only one base pair forms. From the ratio between the colliding rate and the escape rate one gets a weighting probability of $\mathrm{p}_{H y b}=10^{-2}$ for a complete reaction to occur. Therefore, we end with the result that the effective rate of hybridisation is $k_{o n}^{e x p}=\mathrm{p}_{H y b} \times k_{o n}^{\text {diff }}=10^{-2} k_{o n}^{\text {diff }}$, which is a value quite close to the experimental one.

\section{DNA Strand Displacement an Isothermal Ratchet with a Complex Biased Forcing}

\subsection{Single File Problem and Varying Boundary Conditions}

The strand displacement is a one dimensional random walk problem with short range interaction between particles. However, contrary to a common single file problem, there is a possibility that one of the two strands can be ejected from the track. As we may expect, the process of removing a strand has a cost, once an input strand is fixed at the template, the whole system made of the three strands is out of 


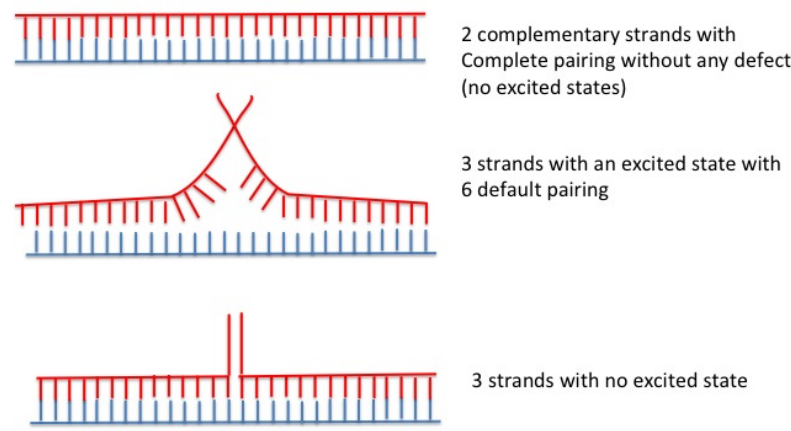

Figure 4. Scheme of the excited states involving two strands on a rigid one-dimensional template (the gate) as it may occur on DNA origamis.

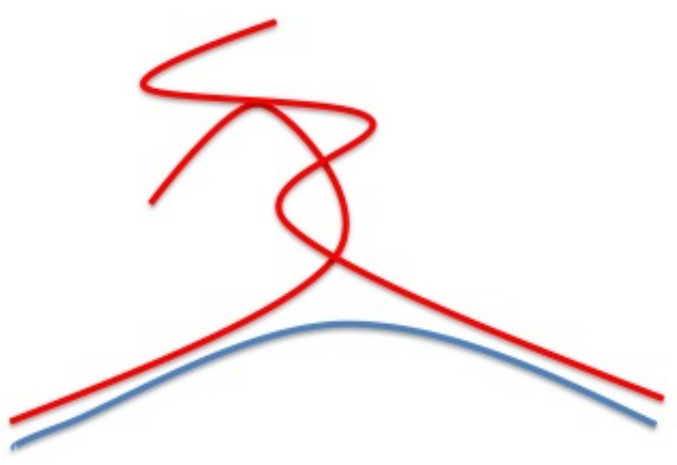

Figure 5. Scheme of the excited states involving two strands on a flexible one-dimensional template (the gate) as it may occur in solution.

equilibrium until one of the two complementary strands has been ejected. In general, the collisions between particles used to be the process that brings back an out of equilibrium state to the equilibrium one. In the present case, a complete different situation arises where the hybridisation of the toe hold sequence forces the two strands to collide each other. The collisions between the two strands generate a large number of configurational states that cannot be enumerate. One way for understanding the main effect of those out of equilibrium configurations is that they change the barrier height controlling the hopping rate between sites. This non trivial statement may be understood another way around, the collisions heat the system such that a few complementary nucleotides reach a continuum of excited states where, for a short time, they are no longer paired together. A simple scheme illustrating the structure of the excited states is shown in figures 4 and 5. Because a great number of configurations can be generated with different consequences on the structure of the excited state, it is a safe assumption to consider a continuous variation of the nucleotides stability with an additional free energy varying from 0 up to $\mathrm{n} U_{l}$, where $\mathrm{n}$ is the number of nucleotides involved in the neighbourhood of the head migration. Note that the probability distribution of those excited states is largely unknown, which forces us to employ such a crude representation to get clues on a possible generic behaviour. Furthermore, we assume those excited states are equally distributed between the two strands so that the structure remains symmetric at the local scale and no biased effects are expected. However, when one of the two ends of the track is reached 
and that different boundary conditions apply, significant changes of the device properties are expected.

Using the FPE [27], we showed how the boundary conditions may have a major effect (see figure 6) on the properties of the device and can be further used to evaluate the different behaviours between tethered devices on DNA origamis and those in diluted solutions. In diluted solution the device can be perfectly symmetric as it happens with the seesaw gates [9], while when the device is tethered on origamis, a highly asymmetric structure is generated through the hybridisation of a fraction of the strands on the origamis [23]. A highly asymmetric structure generates several interesting results, for instance we got the main result that whatever the toe hold strength is the logical operation always succeeds $[27,30]$ (see figure 6).

Asymmetric structures are also obtained in diluted solution when the toe hold is large enough and only set on one side of the gate (figure 2). When the toe hold is long enough it leads to something like a reflecting condition, so that whatever the structure of the excited states, it will not be able to force the input strand to leave the device. On the other side, the end of the output, the excited states will induce a dramatic effect, due to the collisions the output strand may be removed without the need to reach the ultimate, rightmost site (see scheme in figure 7 and the next paragraph).

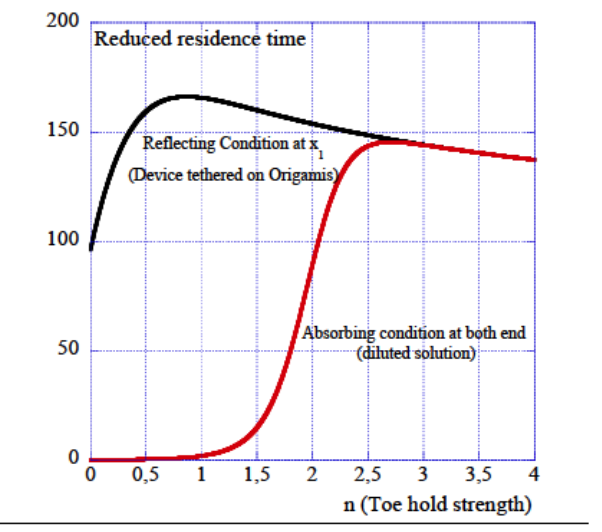

Figure 6. Variation of the escape time as a function of the toe hold strength with for the black curve the reflecting (left side of the device) and absorbing (right side of the device) conditions corresponding to devices tethered on origamis, and for the red curve absorbing condition at both ends corresponding to the case of diluted solution (from [27]). Even with a very weak toehold strength, the DNA nanodevice tethered on a Origami is still able to work.

\subsection{Boundary Conditions Induce the Isothermal Ratchet Functions}

The toe hold length provides a sequence of nucleotides where no competition between strands takes place. This key feature produces an asymmetry of the device, which is described as the result of different boundary conditions. A marked difference between the two ends of the gate occurs when on one side there is a reflecting condition and on the other side an absorbing one (see figure 7). The influence of such a different boundary conditions has to be analysed with the fact that the two strands collide. Let's start with the equation 5 or else the number of trials $\mathrm{M}$ with $\tau=\mathrm{M} \tau_{K}$, from which we calculated $\mathrm{M}=10^{5}-10^{6}$. This simple calculation assumes there is an average trapping time per site of the order of $\tau_{K}$. This approximation is of interest to get an order of magnitude of the total number of jumps M, but is not 
correct. Because the trapping time at any site depends on the colliding configurations between strands, this trapping time is a random variable the distribution of which being independent of the location along the track. Therefore, rather than writing $\tau=\mathrm{M} \tau_{K}$, it is worth writing a more general expression in which no restriction is expressed. The total time after $\mathrm{M}$ jumps is now given by:
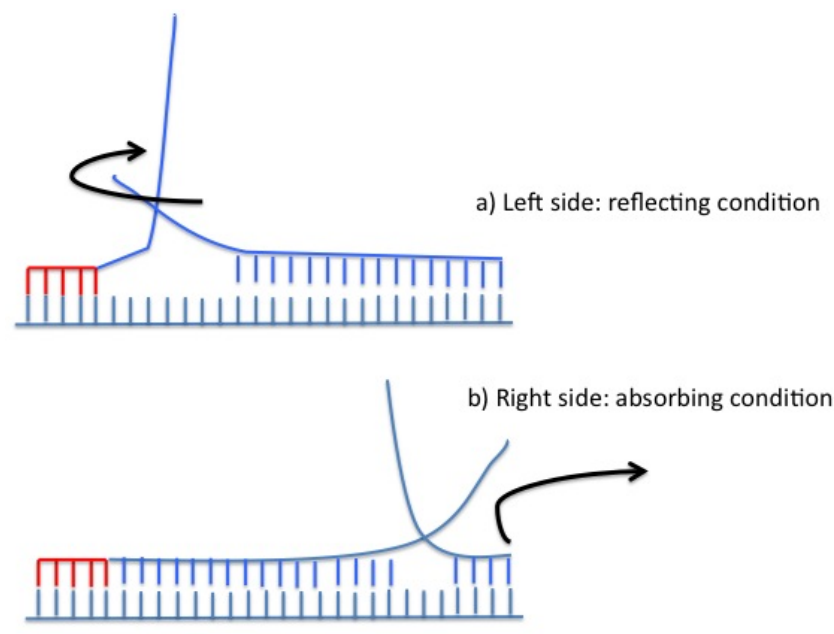

Figure 7. boundary conditions and toe hold (red), the common sequence between input and output strands are blue.

$$
\tau=\sum_{i=1}^{M} \tau_{i}
$$

At any site, the waiting time $\tau_{i}$ is a random variable with a probability distribution $\mathrm{p}\left(\tau_{i}\right)$. The value of the waiting time is no longer related to the hopping rate over a barrier height of a few $k_{B} T$. The waiting time now depends on the interaction between strands and may reach a much higher barrier height decreasing the probability for a jump to succeed (see figure 4 where the colliding states are represented as equivalent to non pairing states). Unfortunately, such a reasoning also remains a crude approximation. The interaction between strands generates a distribution of activation energies that in turn generates the distribution of waiting times and we don't know anything about this distribution of activation energies. The only thing we can say is that they all behave as random variables and that the displacement of the output strand can be reasonably described as the result of a large number of jumps from site to site with uncorrelated random waiting times at each site. This type of disorder with random waiting times at each site also leads to a sub diffusive behaviour which is not unlike the one given by equation 4 [31,32].

In solution, the asymmetry comes from the fact that with a toe hold stable enough, the device is locked at that side (left side in figures 7), from which no strand can escape. Keeping in mind two or three nucleotides would not be able to lock the device, let's take $n=5$ as the lower bound number below which the toe hold strength would not provide an efficient forcing. Then, the strength of the asymmetry can be understood as follow: when the strands move toward the right end side, the stability of the toe hold gives an upper bound time $\tau_{t h}$ to the set of waiting times $\tau_{i}$. Therefore, what we only need is to evaluate the longest waiting time one can have when the two interacting strands run altogether. If this longest waiting time is smaller than the toe hold one $\tau_{t h}$, then the reflecting condition applies and the probability for the output strand to be removed is 1 , keeping in mind it can take quite a long time to reach the exit.

Before we estimate the balance between the two ends of the device, it is worth noting that the collisions not only leads to a sub diffusive behaviour but also produce an entropy that drives the device out of equilibrium. Those collisions reduce the free energy of the complementary strands lowering the stability of the device (something similar to the nucleation of loops decreasing the melting temperature of a double strands). When the two interacting strands are located at the proximity of the right exit side, the 
degradation of the free energy can lead to dramatic effects increasing the speed to sort out the output strand. Say the length of the toe hold is $\mathrm{n}$ and $\alpha$ the number of nucleotides below which the hybridisation is not stable, we are interested in the longest waiting time when the two competing strands are close to the right end, say at a distance $\mathrm{m}$ from the end. At the location $\mathrm{m}$, an upper bound of the residence time of the output strand is given by:

$$
\tau_{m}=\tau_{0} \exp \left[(m-\alpha) U_{l}\right]
$$

This time is much larger than $\tau_{K}$, the first time we derive using the Kramer' description [27]. If $\tau_{m}$ is smaller than $\tau_{t h}=\tau_{0} \exp \left[n U_{l}\right]$, then the output strand is more likely to be removed following a cascade of events. Let's consider a directed motion toward the exit. Because the jump events occur at random, the probability to reach the end of the device is the product of the probabilities per unit time of events to happen at each site. An upper bound of the time required to reach the end, or else the probability for the output to diffuse away from the gate when the head is located at a distance $\mathrm{m}$ from the exit will be :

$$
P_{\text {exit }} \approx\left(\tau_{m}\right)^{-(m-\alpha)}=\tau_{0}^{-(m-\alpha)} \exp \left[-(m-\alpha)^{2} U_{l}\right]
$$

\section{Discussion}

Equations 8 and 9 tell us the migration time can be very long until we reach the proximity of the end of the device where the absorbing condition takes place. Close to the exit, a compressed exponential behaviour can lead to dramatic change since the output strand can diffuse away without the need to reach the ultimate location of the last paired nucleotides. This behaviour is similar to the one observed on the ageing effects of life systems and survival probability giving an estimate of the maximum age a human can reach [33]. It is also similar to the expected non exponential behaviour of the folding of proteins [34]. From the above analysis, the two strands of the DNA nanodevice can be trapped for quite a long time at the beginning and in the middle of the device. The device spends most of the time in moving the strands back and forth until they both reach the proximity of the absorbing end at a time that belongs the tail of the distribution. Then an abrupt change occurs and the output strand diffuses away.

In a recent paper we reported experimental results of DNA computation with DNA nanodevices tethered on origamis [23]. The aim was to amplify an input signal with four output gates and fuel strands. We showed that the devices' yield was low, which was recently confirmed [30,35]. As claimed at the beginning, the use of confinement gives several advantages, however with the toe hold strand displacement technic it rises a major issue upon the interaction between strands. Because the template, the gate output structure, is more or less rigidly fixed on the origamis it may induce a different regime of colliding interactions between strands than those in diluted solution where the template can adopt more flexible conformations [36]. If stronger colliding interactions are more likely to happen when the devices are tethered on origamis, then the time spent on the device, without removing the output, can reach higher values, lowering the gain due to the confinement. In addition, a question rises upon the efficiency of the toe hold hybridisation on origamis. It may as well be weakened, thus decreasing the asymmetry of the reaction path and consequently the efficiency of the device.

\section{Conclusion}

When one wants to conceive a network of Chemical Logical Gates, we need to use chemical species as the outputs that bear the required information. Therefore the strand displacement technique is, at least at this very moment, the best we have in hands to perform a set of logical boolean like operations. An alternative, for instance using loops based on the molecular beacon technic, hardly will be able to produce chemical species we can further use to trigger another action. In this paper we have discussed the basic principles at work when the toe hold strand displacement technique is used. Following this analysis we find that while the use of confined devices would provide a great advantage, it may also lead to a different set of colliding interactions with respect to the ones occurring in diluted solution. In other words, the dissipation of the working device might also be significantly different in confined environment, which may lead to severe restriction we have to take into account when designing devices tethered on Origamis. 
Acknowledgments. VIBBnano ANR Vibbnano [ANR-10-NANO-04-03] Nanobiotech Investment for the Future

\section{References}

1. N. C. Seeman. Nucleic acid junctions and lattices. J. Theor. Biol., 99:237-247, 1982.

2. N. C. Seeman. Nanomaterials based on dna. Annu. Rev. Biochem., 79:65-87, 2010.

3. P. Rothemund. Folding dna to create nanoscale shapes and patterns. Nature, 440:297-302, 2006.

4. B. Yurke, A. Tuberfield, Allen P. Mills, F.C. Simmel, and J.L. Neumann. A dna-fuelled molecular machine made of dna. Nature, 406:605-608, 2000.

5. D. Soloveichik. Dna as a universal substrate for chemical kinetics. Proc. Natl Acad. Sci. USA, 107:5393-5398, 2010.

6. A. J. Turberfield, J. C. Mitchell, B. Yurke, A. P. Mills, M. I. Blakey, and F. C. Simmel. Dna fuel for free-running nanomachines. Phys. Rev. Lett., 90:118102, 2003.

7. F. C. Simmel and B. Yurke. A dna-based molecular device switchable between three distinct mechanical states. Appl. Phys. Lett., 80:883-885, 2002.

8. B. Yurke. Using dna to power nanostructures. Genet. Program. Evolvable Machines, 4:111-122, 2003.

9. L. Qian and E. Winfree. Scaling up digital circuit computation with dna strand dis-placement cascades. Science, 332:1196-1201, 2011.

10. B. Li, A. D. Ellington, and X. Chen. Rational, modular adaptation of enzyme-free dna circuits to multiple detection methods. Nucleic Acids Research, 39:No. 16, e110, 2011.

11. K. Montagne, R. Plasson, Y. Sakai, T. Fujii, and Y. Rondelez. Programming an in vitro dna oscillator using a molecular networking strategy. Molecular Systems Biology, 7:Article number 466, 2011.

12. P. Alberti and J.L. Mergny. Dna duplex-quadruplex exchange as the basis for a nanomolecular machine. Proc. Natl. Acad. Sci., 100:1569-1573, 2003.

13. K. Lund, A. J. Manzo, N. Dabby, N. Michelotti, A. Johnson-Buck, J. Nangreave, S.Taylor, R. Pei, M. N. Stojanovic, N. G. Walter, E. Winfree, and H. Yan. Molecular robots guided by prescriptive landscapes. Nature, 465:206-210, 2010.

14. J. S. Shin and N.A. Pierce. A synthetic dna walker for molecular transport. J. Am. Chem. Soc., 126:1083410835, 2004.

15. P. Yin, H. Yan, A. J. Turberfield X. G. Daniell, and J.H. Reif. A unidirectional dna walker that moves autonomously along a track. Angew. Chem. Int. Ed., 43:4906-4911, 2004.

16. S. F. J. Wickham, J. Bath, Y. Katsuda, M. E. K. Hidaka, H. Sugiyama, and A.J. Turberfield. A dna-based molecular motor that can navigate a network of tracks. Nature Nanotechnology, 7:169-173, 2012.

17. T. Omabegho, R. Sha, and N. C. Seeman. A bipedal dna brownian motor with coordinated legs. Science, 324:67-71, 2009.

18. S.M. Douglas, I. Bachelet, and G.M. Church. A logic-gated nanorobot for targeted transport of molecular payloads. Science, 335:831-834, 2012.

19. E. S. Andersen, M. Dong, M. M. Nielsen, K. Jahn, R. Subramani, W. Mamdouh, M. M. Golas, B. Sander, H. Stark, C. L. P. Oliveira, V. Petersen, J. S. ;and Birkedal, F. Besenbacher, K. V. Gothelf, and J. Kjems. Self-assembly of a nanoscale dna box with a controllable lid. Nature, 459:73-76, 2009.

20. Uri Alon. An introduction to systems biology. Chapman and Hall/CRC press, Taylor and Francis, 2007.

21. William Bialek. Biophysics. searching for principles. Princeton University Press, 2012.

22. Eric R. Kandel, James H. Schwartz, and Thomas M. Jessel. Principles of neural science. McGraw-Hill, Fourth Edition, 2000.

23. Ismael Mullor Ruiz, Jean-Michel Arbona, Amitkumar Lad, Oscar Mendoza, Jean-Pierre Aimé, and Juan Elezgaray. Connecting localized dna strand displacement reactions. Nanoscale, 7:12970, 2015.

24. D. Y. Zhang and E. Winfree. Control of dna strand displacement kinetics using toehold exchange. J. Am. Chem. Soc., 131:17303-17314, 2009.

25. D.Y. Zhang. Dynamic dna strand displacement circuits. Thesis, California Institute of Technology Pasadena, California., May 2010.

26. D.Y. Zhang and G. Seelig. Dynamic dna nanotechnology using strand-displacement reactions. Nat. Chem., 3:103-113, 2011.

27. Jean-Pierre Aimé and Juan Elezgaray. Dna nano devices as a biased random walk process: A case study of isothermal ratchet? Materials Sciences and Applications, 6:401-419, 2015.

28. S. Alexander and P. Pincus. Diffusion of labeled particles on one-dimensional chains. Physical Review B, 18(4):2011-2012, 1978.

29. P Sekhar Burada, Peter Hänggi, Fabio Marchesoni, Gerhard Schmid, and Peter Talkner. Diffusion in confined geometries. ChemPhysChem, 10(1):45-54, 2009. 
30. Oscar Mendoza, Jean-Pierre Aimé, and Juan Elezgaray. Dna computing using strand displacement: Localized amplification circuits (lac) dna origami and diluted solution. submitted, 2016.

31. E. Ben-Naim and P.L. Krapivsky. Enhanced diffusion in disordered systems. Phys. Rev. Lett., 102:190602, 2009.

32. Jean-Philippe Bouchaud and Antoine Georges. Anomalous diffusion in disordered media: Statistical mehcanism, models and physical applications. PHYSICS REPORTS, 195:127-293, 1990.

33. Byung Mook Weon and Jung Ho Je. Theoretical estimation of maximum human lifespan. Biogerontology, 10:65-71, 2009.

34. Masaki Sasai Hironori K. Nakamura and Mitsunori Takano. Scrutinizing the squeezed exponential kinetics observed in the folding simulation of an off-lattice go-like protein model. Chemical Physics, 307:259-267, 2004.

35. Oscar Mendoza, Jean-Louis Mergny, Jean-Pierre Aimé, and Juan Elezgaray. G quadruplexes light up localized dna circuits. Nano Letters, 16:624, 2016.

36. N. Srinivas, T. E. Ouldridge, P. Sulc, J.M. Schaeffer, B. Yurke, A.A. Louis, J. P. K. Doye, and E. Winfree. On the biophysics and kinetics of toehold-mediated dna strand displacement. Nucleic Acids Research, 41:10641-10658, 2013. 